\title{
Semistability of Certain Bundles on a Quintic Calabi-Yau Threefold
}

\author{
Maria Chiara BRAMBILLA
}

\author{
Dipartimento di Matematica e Applicazioni per l'Architettura \\ Università di Firenze \\ piazza Ghiberti, 27 \\ 50122, Florence - Italy \\ brambilla@math.unifi.it
}

Received: January 16, 2008

Accepted: February 11, 2008

\begin{abstract}
In a recent paper Douglas and Zhou aim for explicit examples of string theory compactifications that have a different number of generations and can be connected. For this purpose, they provide a list of bundles on a quintic Calabi-Yau threefold. They need to show that (at least some of) these bundles are semistable and leave this as an open question. In this paper we prove the semistability of most of the bundles in the list, thus completing the result of Douglas and Zhou.
\end{abstract}

Key words: semistability, vector bundles.

2000 Mathematics Subject Classification: 14J60, 14F05.

\section{Introduction}

The study of stable vector bundles on Calabi-Yau threefolds has many applications to physics. In [3] Douglas and Zhou study string theory compactification and illustrate the chirality change with different examples. In particular, in [3, section 3] they consider heterotic string theory with gauge group $E_{8} \times E_{8}$ compactified on a simplyconnected compact Calabi-Yau manifold $M$. They are interested in showing that there exist compactifications on the same Calabi-Yau with different number of generations which can be connected. For this purpose they need to find examples of semistable

The author was partially supported by Italian MIUR funds and by a CNR-NATO Advanced Fellowship. 
holomorphic vector bundles on the Calabi-Yau, whose Chern classes differ only in $c_{3}$. The semistability condition is required in order to get supersymmetric vacua.

In [3, section 3.3] Douglas and Zhou provide a list of bundles $V$ (of rank $3 \leq r \leq 8$ ) on a quintic Calabi-Yau $M \subset \mathbb{P}^{4}$, which satisfy the following conditions:

$$
c_{1}(V)=0, \quad c_{2}(V)=c_{2}(T M), \quad c_{3} \text { arbitrary } .
$$

We recall this list in table 1 below. Then they need to show that these bundles are semistable. In [3, appendix $\mathrm{A}]$ the authors consider one interesting example ( $V_{8}$ in table 1 below), checking the stability with respect to subsheaves that have a similar monad description. Nevertheless they do not give a complete proof even in this case, leaving open the question of semistability.

In this paper we give a proof of the semistability for most of the bundles in the list of Douglas and Zhou. In this way we complete the result of [3]. The precise statement of our result is given in Theorem 2.1 below. In order to prove the semistability, we use different methods in the following three cases: when the rank of the bundle is 4 , when it is 3 and when it is greater than 4 .

More precisely in Proposition 3.1 we show the semistability of the bundles with rank 4 on a general smooth quintic in $\mathbb{P}^{4}$. The proof is based on computations on $\mathbb{P}^{4}$ and on Flenner's theorem. In Proposition 3.3 we prove that all the sheaves with rank 3 in the table, when restricted to a general smooth quintic hypersurface in $\mathbb{P}^{4}$, are stable bundles. In this case we make computations directly on the threefold, to work with locally free sheaves.

For bundles of rank greater than 4 we cannot apply the same argument, but we are able to solve the problem in some cases by using known results on bundles on $\mathbb{P}^{2}$. In Proposition 3.4 we prove the semistability of a general bundle with certain resolution on a general smooth quintic in $\mathbb{P}^{4}$. We obtain this result by restricting to a general plane and by using the Drézet-Le Potier criterion for the existence of a stable bundle on $\mathbb{P}^{2}$. From this argument we deduce the semistability of our bundles when the resolution is general in $\mathbb{P}^{2}$ (that is for bundles $V_{10}, V_{12}, V_{14}, V_{16}$ in table 1 ).

\section{Preliminaries}

Here we collect some useful results on vector bundles on projective varieties. For general references see, e.g., [7] or [9].

Let $\mathbb{P}^{n}$ denote the complex projective space of dimension $n$. Let $X$ be a complex projective manifold with $\operatorname{Pic}(X) \cong \mathbb{Z}$. Given a sheaf $E$ on $X$ we denote its slope by $\mu(E)=\frac{c_{1}(E)}{\mathrm{rk}(E)}$. A bundle $V$ on $X$ is called normalized if $c_{1}(V) \in\{-r+1, \ldots,-1,0\}$, i.e., if $-1<\mu(V) \leq 0$. We denote by $V_{\text {norm }}$ the unique twist of $V$ which is normalized.

A holomorphic vector bundle $V$ on $X$ is called stable if for any coherent subsheaf $S$ of $V$ with $0<\operatorname{rk}(S)<\operatorname{rk}(V)$ we have $\mu(S)<\mu(V)$, and semistable if for any coherent subsheaf $S$ we have $\mu(S) \leq \mu(V)$. This notion of semistability is also called slopesemistability. 
A well known criterion for stability of bundles is the following. Notice that this is not a necessary condition and that the assumption $\operatorname{Pic}(X) \cong \mathbb{Z}$ cannot be omitted.

Proposition 1.1. Let $V$ be a vector bundle on a projective manifold $X$ with $\operatorname{Pic}(X) \cong \mathbb{Z}$. If $\mathrm{H}^{0}\left(X,\left(\bigwedge^{q} V\right)_{\text {norm }}\right)=0$ for any $1 \leq q \leq \operatorname{rk}(V)-1$, then $V$ is stable.

We will use the following technical fact. For more details see, e.g., [5, appendix 2]. Remark 1.2. Any exact sequence of vector bundles

$$
0 \longrightarrow A \longrightarrow B \longrightarrow C \longrightarrow 0
$$

induces the following exact sequence for any $q \geq 1$ :

$$
0 \longrightarrow S^{q} A \longrightarrow S^{q-1} A \otimes B \longrightarrow \cdots \longrightarrow A \otimes \bigwedge^{q-1} B \longrightarrow \bigwedge^{q} B \longrightarrow \bigwedge^{q} C \longrightarrow 0 .
$$

We state Flenner's theorem in the particular case of hypersurfaces in $\mathbb{P}^{n}$. See $[7$, Theorem 7.1.1] for a more general result and a proof.

Theorem 1.3 (Flenner). Assume

$$
\left(\begin{array}{c}
d+n \\
d
\end{array}\right)-d-1>d \max \left\{\frac{r^{2}-1}{4}, 1\right\} .
$$

If $E$ is a semistable sheaf of rank $r$ on $\mathbb{P}^{n}$, then the restriction $\left.E\right|_{X}$ to a general smooth hypersurface $X$ of degree $d$ in $\mathbb{P}^{n}$ is semistable.

Another well known result is the following. For more details see [1, chapter 2].

Proposition 1.4. Let $\phi: E \rightarrow F$ be a morphism of vector bundles on a projective variety of dimension $N$, with $e=\operatorname{rk}(E), f=\operatorname{rk}(F)$ and $e \leq f$. If $E^{*} \otimes F$ is globally generated and $f-e+1>N$, then for a general $\phi \in \operatorname{Hom}(E, F)$ the sheaf $\operatorname{Coker}(\phi)$ is locally free, i.e., is a vector bundle.

The following criterion is a particular case (for $c_{1}=0$ ) of the Drézet-Le Potier theorem (see [4, Theorem C]).

Theorem 1.5 (Drézet-Le Potier). Given $r, c \in \mathbb{Z}$ such that

$$
c \geq r>0
$$

there exists a stable bundle on $\mathbb{P}^{2}$ with rank $r$ and Chern classes $c_{1}=0$ and $c_{2}=c$.

Let $M_{\mathbb{P}^{2}}\left(r, c_{1}, c_{2}\right)$ denote the moduli space of semistable sheaves on $\mathbb{P}^{2}$ of rank $r$ and Chern classes $c_{1}, c_{2}$. It is known that $M_{\mathbb{P}^{2}}\left(r, c_{1}, c_{2}\right)$ is irreducible, and moreover the following result holds (see $[2,6]$ ). 
Proposition 1.6. A general bundle in the space $M_{\mathbb{P}^{2}}\left(r, c_{1}, c_{2}\right)$ has resolution either of the form

$$
0 \longrightarrow \mathcal{O}_{\mathbb{P}^{2}}(k-2)^{a} \oplus \mathcal{O}_{\mathbb{P}^{2}}(k-1)^{b} \longrightarrow \mathcal{O}_{\mathbb{P}^{2}}(k)^{c} \longrightarrow V \longrightarrow 0,
$$

or

$$
0 \longrightarrow \mathcal{O}_{\mathbb{P}^{2}}(k-2)^{d} \longrightarrow \mathcal{O}_{\mathbb{P}^{2}}(k-1)^{e} \oplus \mathcal{O}_{\mathbb{P}^{2}}(k)^{f} \longrightarrow V \longrightarrow 0,
$$

for some $k \in \mathbb{Z}, a, b \geq 0$, and $c>0$, or $d, e \geq 0$ and $f>0$.

Remark 1.7. It is easy to see that given any triple $\left(r, c_{1}, c_{2}\right)$, there exists a unique triple either $(a, b, c)$, or $(d, e, f)$ such that a bundle $V$ with resolution either (1), or (2) has rank $r$ and Chern classes $c_{1}, c_{2}$.

Remark 1.8. Recall that the subset of stable bundles in the space of bundles with fixed resolution either (1), or (2) is open. This implies that if there exists a stable bundle in the moduli space $M_{\mathbb{P}^{2}}\left(r, c_{1}, c_{2}\right)$, then a general bundle with the corresponding general resolution is stable.

Finally we recall that by a well known Maruyama's result (see [8]) stability and semistability are open properties.

\section{Bundles on the quintic}

In [3, section 3] the authors perform an explicit search of bundles on a quintic CalabiYau $M \subset \mathbb{P}^{4}$, whose invariants satisfy suitable conditions. Here we recall briefly the construction of such bundles.

Let $m_{j}$ for $j=1, \ldots, m$ and $n_{i}$ for $i=1, \ldots, m+r$ be integers such that $m_{j} \geq n_{i}$ for any $i, j$. Consider a general map

$$
\phi: \bigoplus_{i=1}^{r+m} \mathcal{O}_{\mathbb{P}^{4}}\left(n_{i}\right) \longrightarrow \bigoplus_{j=1}^{m} \mathcal{O}_{\mathbb{P}^{4}}\left(m_{j}\right),
$$

which can be represented by a $m \times(r+m)$ matrix of homogeneous polynomials $Q_{i}^{j}$ of degree $m_{j}-n_{i}$. Denoting by $V$ the sheaf $\operatorname{ker}(\phi)$ we obtain the following exact sequence on $\mathbb{P}^{4}$ :

$$
0 \longrightarrow V \longrightarrow \bigoplus_{i=1}^{r+m} \mathcal{O}_{\mathbb{P}^{4}}\left(n_{i}\right) \stackrel{\phi}{\longrightarrow} \bigoplus_{j=1}^{m} \mathcal{O}_{\mathbb{P}^{4}}\left(m_{j}\right)
$$

Notice that if $r \geq 4$ then the general map $\phi$ is surjective and $V$ is locally free on $\mathbb{P}^{4}$, by Proposition 1.4. This is not true in the case $r=3$. Indeed it is easy to check that, for a general choice of $n_{i}$ and $m_{j}$, we have $c_{4}(V) \neq 0$ and this implies that the 
Table 1

\begin{tabular}{|c|c|c|c|}
\hline & rank & $\left(n_{i}\right)$ & $\left(m_{j}\right)$ \\
\hline \hline$V_{1}$ & 3 & $(22222222)$ & $(33334)$ \\
\hline$V_{2}$ & 3 & $(122222)$ & $(344)$ \\
\hline$V_{3}$ & 3 & $(112233)$ & $(444)$ \\
\hline$V_{4}$ & 3 & $(11222)$ & $(35)$ \\
\hline$V_{5}$ & 3 & $(11133)$ & $(45)$ \\
\hline$V_{6}$ & 4 & $(1122222222)$ & $(333333)$ \\
\hline$V_{7}$ & 4 & $(11122222)$ & $(3334)$ \\
\hline$V_{8}$ & 4 & $(111122)$ & $(44)$ \\
\hline$V_{9}$ & 4 & $(11111)$ & $(5)$ \\
\hline$V_{10}$ & 5 & $(1111122222)$ & $(33333)$ \\
\hline$V_{11}$ & 5 & $(11111122)$ & $(334)$ \\
\hline$V_{12}$ & 6 & $(1111111122)$ & $(3333)$ \\
\hline$V_{13}$ & 6 & $(111111111)$ & $(234)$ \\
\hline$V_{14}$ & 7 & $(11111111111)$ & $(2333)$ \\
\hline$V_{15}$ & 7 & $(11111111111)$ & $(22224)$ \\
\hline$V_{16}$ & 8 & $(111111111111)$ & $(222233)$ \\
\hline
\end{tabular}

rank 3 sheaf $V$ is not locally free. On the other hand, if $r=3$, given a general quintic threefold $M$ in $\mathbb{P}^{4}$, we have that the restriction of the general map $\phi$ to $M$

$$
\phi_{\mid M}: \bigoplus_{i=1}^{r+m} \mathcal{O}_{M}\left(n_{i}\right) \longrightarrow \bigoplus_{j=1}^{m} \mathcal{O}_{M}\left(m_{j}\right)
$$

is surjective and the restriction of $V$ to $M$ is locally free, by Proposition 1.4.

Imposing that $c_{1}(V)=0$ and $c_{2}(V)=c_{2}(T M)=10$, one can easily list the possible values of $n_{i}$ and $m_{j}$. In table 1 we recall the list provided in [3]. Observe that the third Chern class is not fixed.

For $1 \leq k \leq 16$, we say that $V$ is a general sheaf of type $V_{k}$ if it fits in the exact sequence (3) for a general $\phi$ and for the corresponding values of $n_{i}$ and $m_{j}$ in the table.

In their paper Douglas and Zhou are interested in proving the (semi)stability of the restriction of sheaves of type $V_{k}$ to a general quintic $M$ in $\mathbb{P}^{4}$. Here we state our result, and we prove it in the following section.

Theorem 2.1. Let $V$ be a general sheaf of type $V_{k}$.

- If $k \in\{1, \ldots, 5\}$, then the restriction of $V$ to a general smooth quintic hypersurface in $\mathbb{P}^{4}$ is a stable bundle. 
- If $k \in\{6, \ldots, 10,12,14,16\}$, then the restriction of $V$ to a general smooth quintic hypersurface in $\mathbb{P}^{4}$ is a semistable bundle.

\section{Proof of semistability}

In this section we give the proof of Theorem 2.1, splitting it in three parts. First of all we consider the case of rank 4 bundles.

Proposition 3.1. Let $V$ be a general bundle of type $V_{k}$ for $6 \leq k \leq 9$. Then $V$ is semistable on a general smooth quintic hypersurface in $\mathbb{P}^{4}$.

Before giving the proof of the previous proposition, let us consider in detail the case of a bundle of type $V_{8}$ (which is the example studied by Douglas and Zhou in [3]). In the following we make standard computations, nevertheless we explain the details for the reader's convenience.

Example 3.2. Let $V$ be a general bundle of type $V_{8}$. Since (semi)stability is invariant up to duality, we will check the (semi)stability of the dual bundle $E=V^{*}$, which has the following resolution on $\mathbb{P}^{4}$ :

$$
0 \longrightarrow \mathcal{O}_{\mathbb{P}^{4}}(-4)^{2} \longrightarrow \mathcal{O}_{\mathbb{P}^{4}}(-2)^{2} \oplus \mathcal{O}_{\mathbb{P}^{4}}(-1)^{4} \longrightarrow E \longrightarrow 0 .
$$

To check the stability of $E$, by Proposition 1.1, we need to show that $\mathrm{H}^{0}\left(\mathbb{P}^{4},\left(\bigwedge^{q} E\right)_{\text {norm }}\right)=0$ for any $1 \leq q \leq 3$. Since $c_{1}(E)=0$, it is obvious that $\left(\bigwedge^{q} E\right)_{\text {norm }}=\bigwedge^{q} E$ for any $q$. Then we have to prove that

$$
\mathrm{H}^{0}\left(\mathbb{P}^{4}, E\right)=0, \quad \mathrm{H}^{0}\left(\mathbb{P}^{4}, \bigwedge^{2} E\right)=0, \quad \mathrm{H}^{0}\left(\mathbb{P}^{4}, \bigwedge^{3} E\right)=0 .
$$

By the cohomology sequence associated to (4) we immediately get the first vanishing. Indeed by Remark 1.2 we can compute the following resolution for $\bigwedge^{2} E$

$$
\begin{aligned}
0 & \longrightarrow \mathcal{O}_{\mathbb{P}^{4}}(-8)^{3} \longrightarrow \mathcal{O}_{\mathbb{P}^{4}}(-6)^{4} \oplus \mathcal{O}_{\mathbb{P}^{4}}(-5)^{8} \\
& \longrightarrow \mathcal{O}_{\mathbb{P}^{4}}(-4) \oplus \mathcal{O}_{\mathbb{P}^{4}}(-3)^{8} \oplus \mathcal{O}_{\mathbb{P}^{4}}(-2)^{6} \longrightarrow \bigwedge^{2} E 0,
\end{aligned}
$$

and, for $\bigwedge^{3} E$,

$$
\begin{aligned}
0 & \longrightarrow \mathcal{O}_{\mathbb{P}^{4}}(-12)^{4} \longrightarrow \mathcal{O}_{\mathbb{P}^{4}}(-10)^{6} \oplus \mathcal{O}_{\mathbb{P}^{4}}(-9)^{12} \\
& \longrightarrow \mathcal{O}_{\mathbb{P}^{4}}(-8)^{2} \oplus \mathcal{O}_{\mathbb{P}^{4}}(-7)^{16} \oplus \mathcal{O}_{\mathbb{P}^{4}}(-6)^{12} \\
& \longrightarrow \mathcal{O}_{\mathbb{P}^{4}}(-5)^{4} \oplus \mathcal{O}_{\mathbb{P}^{4}}(-4)^{12} \oplus \mathcal{O}_{\mathbb{P}^{4}}(-3)^{4} \longrightarrow \bigwedge^{3} E \longrightarrow 0
\end{aligned}
$$

From the resolution of $\bigwedge^{2} E$ we get the following two short exact sequences:

$$
\begin{gathered}
0 \longrightarrow \mathcal{O}_{\mathbb{P}^{4}}(-8)^{3} \longrightarrow \mathcal{O}_{\mathbb{P}^{4}}(-6)^{4} \oplus \mathcal{O}_{\mathbb{P}^{4}}(-5)^{8} \longrightarrow K_{0} \longrightarrow 0, \\
0 \longrightarrow K_{0} \longrightarrow \mathcal{O}_{\mathbb{P}^{4}}(-4) \oplus \mathcal{O}_{\mathbb{P}^{4}}(-3)^{8} \oplus \mathcal{O}_{\mathbb{P}^{4}}(-2)^{6} \longrightarrow \bigwedge^{2} E \longrightarrow 0,
\end{gathered}
$$


and since $\mathrm{H}^{1}\left(\mathbb{P}^{4}, K_{0}\right)=0$, we get $\mathrm{H}^{0}\left(\mathbb{P}^{4}, \bigwedge^{2} E\right)=0$. Analogously from the resolution of $\bigwedge^{3} E$ we get

$$
\begin{gathered}
0 \longrightarrow \mathcal{O}_{\mathbb{P}^{4}}(-12)^{4} \longrightarrow \mathcal{O}_{\mathbb{P}^{4}}(-10)^{6} \oplus \mathcal{O}_{\mathbb{P}^{4}}(-9)^{12} \longrightarrow K_{1} \longrightarrow 0, \\
0 \longrightarrow K_{1} \longrightarrow \mathcal{O}_{\mathbb{P}^{4}}(-8)^{2} \oplus \mathcal{O}_{\mathbb{P}^{4}}(-7)^{16} \oplus \mathcal{O}_{\mathbb{P}^{4}}(-6)^{12} \longrightarrow K_{2} \longrightarrow 0 \\
0 \longrightarrow K_{2} \longrightarrow \mathcal{O}_{\mathbb{P}^{4}}(-5)^{4} \oplus \mathcal{O}_{\mathbb{P}^{4}}(-4)^{12} \oplus \mathcal{O}_{\mathbb{P}^{4}}(-3)^{4} \longrightarrow \bigwedge^{3} E \longrightarrow 0,
\end{gathered}
$$

from which we obtain $\mathrm{H}^{2}\left(\mathbb{P}^{4}, K_{1}\right)=0, \mathrm{H}^{1}\left(\mathbb{P}^{4}, K_{2}\right)=0$, and $\mathrm{H}^{0}\left(\mathbb{P}^{4}, \bigwedge^{3} E\right)=0$. Hence we get

$$
\mathrm{H}^{0}\left(\mathbb{P}^{4}, E\right)=\mathrm{H}^{0}\left(\mathbb{P}^{4}, \bigwedge^{2} E\right)=\mathrm{H}^{0}\left(\mathbb{P}^{4}, \bigwedge^{3} E\right)=0,
$$

which implies that $E$ is stable on $\mathbb{P}^{4}$.

Now from Flenner's theorem it follows that the restriction of $E$ to a general hypersurface of degree $d \geq 2$ is semistable, in particular the restriction of $E$ to a general smooth quintic hypersurface in $\mathbb{P}^{4}$ is semistable.

Finally this implies that $V$ is semistable when restricted to a general smooth quintic hypersurface.

Proof of Proposition 3.1. It is easy to check that the argument used in Example 3.2 holds also for the bundles of type $V_{6}, V_{7}$, and $V_{9}$. Hence all the bundles with rank 4 in table 1 are semistable on a general smooth quintic.

We consider now the case of rank 3 bundles. Recall that such bundles are not locally free on $\mathbb{P}^{4}$, hence the argument used in Example 3.2 does not hold. In this case we work directly on the quintic $M$ and we get the following result.

Proposition 3.3. Let $V$ be a general sheaf of type $V_{k}$ for $1 \leq k \leq 5$. Then the restriction of $V$ to a general smooth quintic hypersurface in $\mathbb{P}^{4}$ is a stable bundle.

Proof. Given a general quintic $M$ in $\mathbb{P}^{4}$, the restriction $\left.V\right|_{M}$ is locally free, by Proposition 1.4. Let $\left.E\right|_{M}$ denote the dual of $\left.V\right|_{M}$. If for example $k=5$, then the bundle $\left.E\right|_{M}$ has the following resolution:

$$
\left.0 \longrightarrow \mathcal{O}_{M}(-5) \oplus \mathcal{O}_{M}(-4) \longrightarrow \mathcal{O}_{M}(-3)^{2} \oplus \mathcal{O}_{M}(-1)^{3} \longrightarrow E\right|_{M} \longrightarrow 0 .
$$

Now we want to apply Proposition 1.1 to $\left.E\right|_{M}$. First of all, computing the cohomology of $\mathcal{O}_{M}(-k)$ from the following exact sequence:

$$
0 \longrightarrow \mathcal{O}_{\mathbb{P}^{4}}(-5) \longrightarrow \mathcal{O}_{\mathbb{P}^{4}} \longrightarrow \mathcal{O}_{M} \longrightarrow 0
$$

we easily obtain $\mathrm{H}^{0}\left(M,\left.E\right|_{M}\right)=0$. On the other hand, by Remark 1.2 we compute the resolution of $\bigwedge^{2}\left(\left.E\right|_{M}\right)$ and we get $\mathrm{H}^{0}\left(M, \bigwedge^{2}\left(\left.E\right|_{M}\right)\right)=0$. Analogously it is easy to check that

$$
\mathrm{H}^{0}\left(M,\left.E\right|_{M}\right)=\mathrm{H}^{0}\left(M, \bigwedge^{2}\left(\left.E\right|_{M}\right)\right)=0
$$


for all cases $V_{1}, \ldots, V_{5}$. Hence the bundle $\left.E\right|_{M}$ is stable on $M$. We conclude that every sheaf with rank 3 of table 1 restricted to a general smooth quintic in $\mathbb{P}^{4}$ is a stable bundle.

Finally we deal with the case of bundles with rank greater than 4 . We stress that in general it is very difficult to prove the stability of bundles with high rank. Nevertheless we are able to prove the semistability of general bundles of type $V_{k}$ for $k \in\{10,12,14,16\}$. The key argument consists in restricting a bundle on $\mathbb{P}^{4}$ to a general $\mathbb{P}^{2} \subset \mathbb{P}^{4}$, and applying the results on bundles on $\mathbb{P}^{2}$ recalled at the end of section 2. We obtain the following result.

Proposition 3.4. Let $V$ be a general bundle of type $V_{k}$, for $k=10,12,14,16$. Then $V$ is semistable on a general smooth quintic in $\mathbb{P}^{4}$.

Proof. Let us show first that $E=V^{*}$ is stable on $\mathbb{P}^{4}$. In order to do this, it suffices to prove that the restriction of $E$ to a general plane is stable.

The restriction $\left.E\right|_{\Pi}$ to a plane $\Pi \subset \mathbb{P}^{4}$ has a resolution either of the form

$$
\left.0 \longrightarrow \mathcal{O}_{\Pi}(-3)^{a} \oplus \mathcal{O}_{\Pi}(-2)^{b} \stackrel{\psi}{\longrightarrow} \mathcal{O}_{\Pi}(-1)^{c} \longrightarrow E\right|_{\Pi} \longrightarrow 0
$$

or

$$
\left.0 \longrightarrow \mathcal{O}_{\Pi}(-3)^{a} \stackrel{\psi}{\longrightarrow} \mathcal{O}_{\Pi}(-2)^{b} \oplus \mathcal{O}_{\Pi}(-1)^{c} \longrightarrow E\right|_{\Pi} \longrightarrow 0
$$

where $a, b, c \in \mathbb{N}$ are given by table 1 and the map $\psi$ is the restriction to $\Pi$ of the transpose $\phi^{\top}$ of the map $\phi$ in (3). Clearly we have that $\psi$ is a general map, since it is the restriction of a general map.

On the other hand, by Proposition 1.6 and Remark 1.7, it follows that a general bundle in $M_{\mathbb{P}^{2}}(5,0,10)$ has resolution of the form $V_{10}$. Analogously a general bundle in $M_{\mathbb{P}^{2}}(6,0,10), M_{\mathbb{P}^{2}}(7,0,10), M_{\mathbb{P}^{2}}(8,0,10)$, respectively, has a resolution of the form $V_{12}, V_{14}, V_{16}$, respectively.

Moreover, we know from Theorem 1.5 that there exists a stable bundle on $\mathbb{P}^{2}$ with $c_{1}=0, c_{2}=10$, and $r \leq 10$. Hence the irreducible space $M_{\mathbb{P}^{2}}(r, 0,10)$ is not empty for $5 \leq r \leq 8$. Therefore from Remark 1.8 it follows that the bundle $\left.E\right|_{\Pi}$ is stable on the plane and $E$ is stable on $\mathbb{P}^{4}$.

Finally from Flenner's theorem it follows that if $E$ is a semistable bundle of rank $5 \leq r \leq 8$ on $\mathbb{P}^{4}$, then its restriction to a general hypersurface of degree 5 is semistable. Hence we conclude that the restriction of $E$ to a general smooth quintic hypersurface in $\mathbb{P}^{4}$ is semistable.

Acknowledgements. The author thanks Adrian Langer for suggesting this problem and for useful discussions, and Giorgio Ottaviani for helpful comments. 


\section{References}

[1] E. Arbarello, M. Cornalba, P. A. Griffiths, and J. Harris, Geometry of algebraic curves, I, Grundlehren der Mathematischen Wissenschaften, vol. 267, Springer-Verlag, New York, 1985.

[2] C. Dionisi and M. Maggesi, Minimal resolution of general stable rank-2 vector bundles on $\mathbb{P}^{2}$, Boll. Unione Mat. Ital. Sez. B Artic. Ric. Mat. (8) 6 (2003), no. 1, 151-160, available at math. $\mathrm{AG} / 0002169$.

[3] M. R. Douglas and C. Zhou, Chirality change in string theory, J. High Energy Phys. 6 (2004), 014, 42 pp. (electronic).

[4] J.-M. Drezet and J. Le Potier, Fibrés stables et fibrés exceptionnels sur $\mathbf{P}_{2}$, Ann. Sci. École Norm. Sup. (4) 18 (1985), no. 2, 193-243 (French, with English summary).

[5] D. Eisenbud, Commutative algebra - with a view toward algebraic geometry, Graduate Texts in Mathematics, vol. 150, Springer-Verlag, New York, 1995.

[6] A. Hirschowitz and Y. Laszlo, Fibrés génériques sur le plan projectif, Math. Ann. 297 (1993), no. $1,85-102$.

[7] D. Huybrechts and M. Lehn, The geometry of moduli spaces of sheaves, Aspects of Mathematics, vol. 31, Friedr. Vieweg \& Sohn, Braunschweig, 1997.

[8] M. Maruyama, Openness of a family of torsion free sheaves, J. Math. Kyoto Univ. 16 (1976), no. $3,627-637$.

[9] C. Okonek, M. Schneider, and H. Spindler, Vector bundles on complex projective spaces, Progress in Mathematics, vol. 3, Birkhäuser, Boston, Mass., 1980. 